\title{
Continuous ERECTOR SPINAE PLANE BLOCK: first choice in perioperative analgesia in thoracotomy surgery?
}

\author{
C. Pinto ${ }^{1} ; \underline{\text { F. Matias }^{1}}$
}

1- Department of Anaesthesia, Centro Hospitalar e Universitário de Coimbra, Coimbra, Portugal

Email: franciscomatias7@Hotmail.com

\section{Backgroud and Aims}

Thoracic epidural analgesia are currently the first line techniques for use in managing perioperative pain follow thoracotomy (1).

Regional anesthetic techniques are strongly recommended primarily to reduce opioid use and the related adverse effects, including hypoventilation, sedation, nausea, and vomiting (2).

In this way, continuous erector spinae plane (ESP) block is growing popularity because of its simplicity, safe and lesser side effects $(1,2)$.

We aim to present the result of the analgesic efficacy of continuous ultrasound-guided ESP block in two cases of thoracotomy surgery.

\section{Case Report:}

65-year-old woman, presented for right inferior lobectomy (adenocarcinoma) and a 49-year-old woman, presented for left superior lobectomy (adenocarcinoma). Combined anesthesia (Balanced general anesthesia + continuous ESP) was performed.

Before induction, the ultrasound-guided unilateral continuous ESP block was performed at the level of T5. 30 milliliters of ropivacaine $0,375 \%$ were administered.

The multimodal approach for postoperative analgesia was: programmed intermittent mandatory boluses through the ESP catheter of ropivacaine $0,2 \% 8 \mathrm{ml} / \mathrm{h}+$

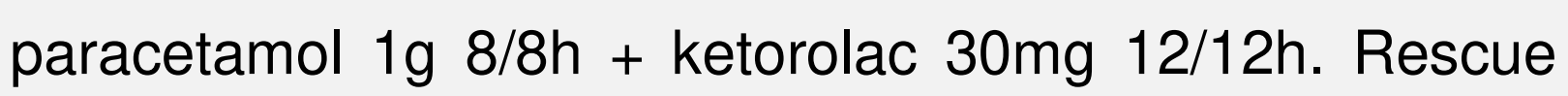
analgesia with tramadol $100 \mathrm{mg}$.

\section{Conclusion}

Pain after thoracotomy can be difficult to control with a multimodal analgesia. Furthermor, thoracic epidural analgesia has potentially serious complications. Recently, ESP block has increased acceptance because it is a safe thoracic block with minor complications. With this two cases authors had shown that continuous ESP block provides effective post-operative analgesia follow thoracotomy with excellent pain control inside multimodal analgesic program with low opioids requirements.

1- Forero M, Adhikary SD, Lopez H, et al. The Erector Spinae Plane Block: A novel analgesic technique in thoracic neuropatic pain. Regional Anesthesia and Pain Medicine. 2016 Sep - Oct 41; 5: 621-27.

2 - Forrero M, Rajarathinam M, Adhikary S, et Chin KJ. Continuous Erector Spinae Plane Block for Rescue Analgesia in Thoracotomy After Epidural Failure: A Case Report. . A \& A Case Reports. MAY 15TH, 2017; 8(10):254-256.
The worst pain was 4 (numeric scale) 3 and 5 hours after surgery, respectively. At this time they both do tramadol 100mg.

Beside this they don't need more rescue analgesia in the first 48 hours.

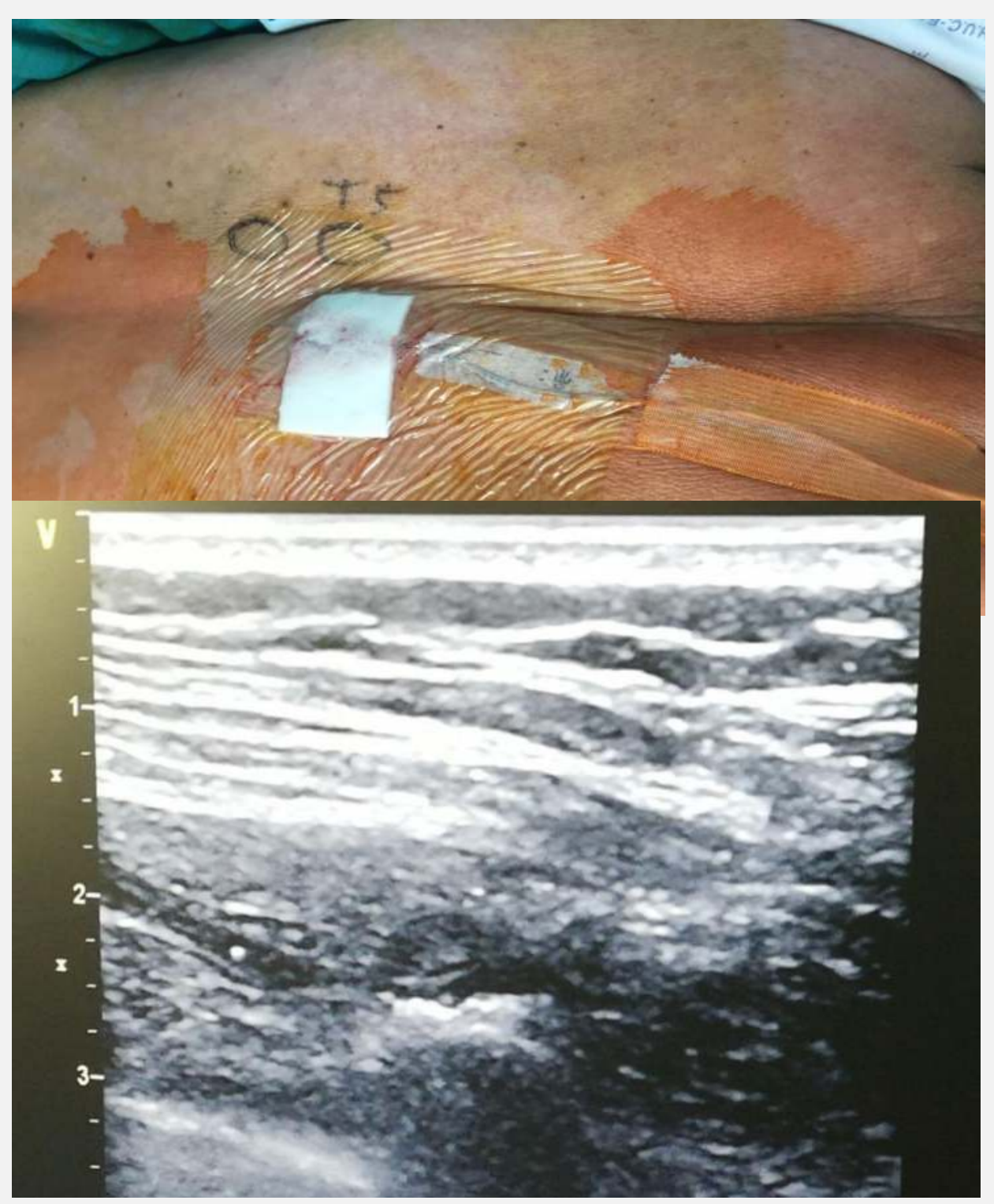

Picture 1: Continuous ESP block

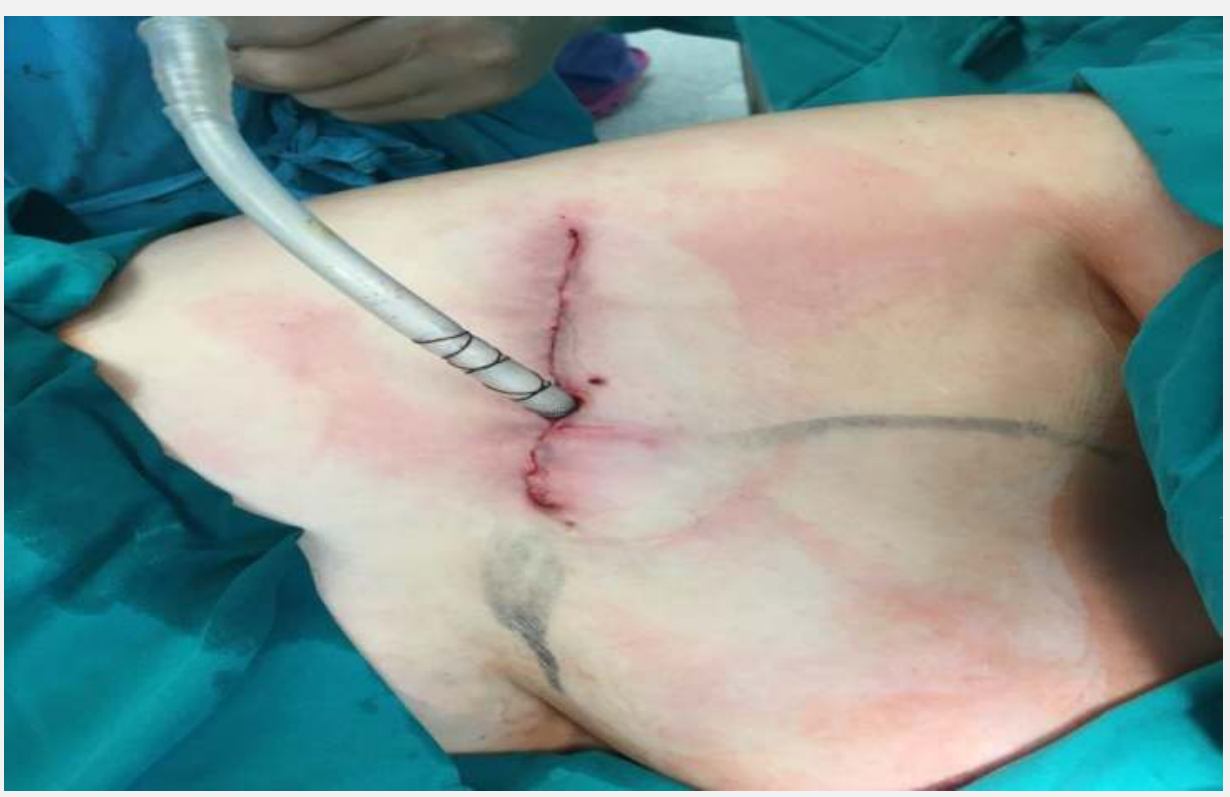

Picture 2: Surgical incision 